Am J Nephrol 2020;51:786-796

DOI: $10.1159 / 000511160$
Received: May 18, 2020

Accepted: August 24, 2020

Published online: October 2, 2020

\title{
Acute Kidney Injury in COVID-19 \\ Patients: An Inner City Hospital Experience and Policy Implications
}

\author{
Umar Zahid $^{\mathrm{a}}$ Preethi Ramachandran $^{\mathrm{b}}$ Samuel Spitalewitz ${ }^{\mathrm{a}}$ Lutfi Alasadi $^{\mathrm{a}}$ \\ Abhishek Chakraborti ${ }^{c}$ Muhammad Azhar ${ }^{\mathrm{a}}$ Galina Mikhalina ${ }^{\mathrm{a}}$ \\ Andleeb Sherazi $^{\mathrm{a}} \quad$ Joshua Tetteh Narh ${ }^{c}$ Pallavi Khattar ${ }^{\mathrm{d}}$ Puneet Bedi ${ }^{\mathrm{a}}$ \\ aDivision of Nephrology, Brookdale University Hospital and Medical Center, Brooklyn, NY, USA; ${ }^{b}$ Division of \\ Hematology/Oncology, Brookdale University Hospital and Medical Center, Brooklyn, NY, USA; 'Department of \\ Internal Medicine, Brookdale University Hospital and Medical Center, Brooklyn, NY, USA; dDepartment of Pathology, \\ Icahn School of Medicine at Mount Sinai, New York, NY, USA
}

\section{Keywords}

Acute kidney injury · Acute respiratory distress syndrome .

Coronavirus disease $19 \cdot$ In-hospital mortality

\begin{abstract}
Background: Although diffuse alveolar damage and respiratory failure are the key features of coronavirus disease 2019 (COVID-19), the involvement of other organs such as the kidney has also been reported. The reports of the incidence of acute kidney injury (AKI) in COVID-19 patients vary widely. In this study, we report our unique experience with AKI in COVID-19 patients in a low socioeconomic and predominantly ethnic minority group and provide its incidence, risk factors, and prognosis to expand the current understanding of this complication. Methods: In this single-center, retrospective cohort study, we analyzed the data of 469 COVID-19 patients admitted to the Brookdale University Hospital in Brooklyn, NY, from March 18 through April 23, 2020. Information regarding demographics, comorbidities, medications, clinical and laboratory data, and outcomes was collected from the electronic medical records. Both univariate and multivariate analyses were performed to determine the association of AKI with in-hospital mortality. Results: The median age was 66
\end{abstract}

years (interquartile range [IQR] 25-75; range 19-101 years), and 268 (57.14\%) patients were male. Estimated glomerular filtration rate (eGFR) as determined by the Modification of Diet in Renal Disease Study Equation was low $(<60 \mathrm{~mL} /$ $\mathrm{min} / 1.73 \mathrm{~m}^{2}$ ) in 207 (44.1\%) patients. During hospitalization, $128(27.3 \%)$ patients developed AKI, and the incidence was significantly higher in those patients presenting with a low eGFR $(N=81,39.1 \% ; p<0.001)$. Male sex, hypertension, the use of angiotensin-converting enzyme inhibitors and nonsteroidal anti-inflammatories, hemodynamic instability, mechanical ventilation, acute respiratory distress syndrome, and admission elevated ferritin, creatinine kinase, brain natriuretic peptide, and troponin 1 were identified as the risk factors for in-hospital AKI. Ninety-seven $(28.45 \%)$ patients died in the non-AKI group versus 91 (71.1\%) in the AKI group $(p<0.001)$. The Cox proportional hazard model after adjusting for age, gender, comorbidities, hemodynamic status, and $\mathrm{PF}$ ratio (arterial oxygen partial pressure $\left[\mathrm{PaO}_{2}\right] /$ fractional inspired oxygen $\left[\mathrm{FiO}_{2}\right]$ ) determined that on admission, an elevated blood urea nitrogen (hazard ratio [HR]: 1.75; 95\% confidence interval $[\mathrm{Cl}] 1.23-2.48)$, a low eGFR (HR 1.43; $\mathrm{Cl}$ 1.1-2.03), AKI stage 1 (HR 1.14; Cl 0.64-2.03), AKI stage 2 (HR $1.86 ; \mathrm{Cl} 1.03-3.56)$, and AKI stage 3 (HR 2.1; $\mathrm{Cl} 1.3-2.81$ ) were independent risk factors for in-hospital mortality. Renal re- karger@karger.com

www.karger.com/ajn

Karger ${ }^{\prime}=$

$$
\text { 少 }
$$

(c) 2020 S. Karger AG, Base
Puneet Bedi

Division of Nephrology, Brookdale University Hospital and Medical Center 1 Brookdale Plaza

Brooklyn, NY 11212 (USA)

pbedi@bhmcny.org 
placement therapy (RRT) did not improve survival in stage III AKI. Conclusion: AKI in our hospitalized COVID-19 patients was common and carried a high mortality, especially in patients with AKI stage 3. RRT did not improve survival. Policy changes and planning for this high incidence of AKI in COVID-19 patients and its associated high mortality are necessary at the local and national levels. @2020 S. Karger AG, Basel

\section{Introduction}

Coronavirus disease 2019 (COVID-19) is associated with a high morbidity and mortality with a disproportionately poor outcome in the minority and low socioeconomic groups [1-3]. As reported by some, the association of acute kidney injury (AKI) and COVID-19 has a high mortality [4]. However, the incidence of reported AKI associated with COVID-19 varies widely [4-9]. It would be expected that kidney involvement is frequent since the virus enters the cell through the angiotensin-converting enzyme 2 (ACE2), which is expressed, in addition to pulmonary type 2 alveolar cells, on renal proximal tubular cells, glomerular visceral and parietal epithelium, and the cytoplasm of the distal tubules and collecting ducts [1013]. In addition, COVID-19 induces hemodynamic instability and severe inflammation, which, in addition to direct renal infection, can induce acute tubular necrosis (ATN). We report our experience with AKI in COVID-19 patients in an inner city, predominantly minority, and a low socioeconomic group and compare our experience with other studies in significantly different cohorts of patients. We identify the incidence, risk factors, and prognosis of AKI and thus provide a broader understanding of this complication in a group of patients not specifically reported upon to date.

\section{Methods}

Study Design and Participants

All adult patients $>18$ years of age admitted to the Brookdale University Hospital and Medical Center with COVID-19 infection from March 18 through April 23, 2020, were studied in this retrospective analysis done in a single medical center serving a low-income minority population in Brooklyn, NY. It was one of the single centers with the most admissions of COVID-19 patients. COVID-19 infection was diagnosed based on clinical presentation, radiographic lung abnormalities, and a positive result of real time PCR. We excluded COVID-19 patients from our study if they were on maintenance hemodialysis or were renal transplant recipients. This study was approved by the Institutional Review Board of
Brookdale Hospital and did not require patient consent because of its retrospective design.

\section{Data Source}

We collected the demographics, the presenting clinical symptoms when available, prior medical history including outpatient medications and prior level of renal function, comorbidities, and in-hospital laboratory data from the electronic medical record (Epic Hyperspace, 2015). Laboratory data consisted of complete blood count, hemostasis parameters, renal and liver function, PF ratio (arterial oxygen partial pressure $\left[\mathrm{PaO}_{2}\right] /$ fractional inspired oxygen $\left[\mathrm{FiO}_{2}\right]$ ), creatinine kinase, lactate dehydrogenase, and inflammatory markers including high-sensitivity C-reactive protein (hs-CRP), ferritin, and D-dimer. Normal range of these measures was provided by our laboratory.

The Kidney Disease: Improving Global Outcome (KDIGO) [14] definition was used to identify AKI. Estimated glomerular filtration rate (eGFR) on admission was taken as baseline eGFR and was divided into $<60$ and $\geq 60 \mathrm{~mL} / \mathrm{min} / 1.73 \mathrm{~m}^{2}$. eGFR was estimated using the Modification of Diet in Renal Disease Study (MDRD) equation. The earliest day of the serum creatinine change that met the KDIGO criteria for AKI was selected as day 1 of AKI. The peak serum creatinine value was used to determine the stage of AKI, with an increase in the serum creatinine of 1.5-1.9, 2.0-2.9, and $>3$ times the baseline serum creatinine defined as AKI stages 1,2 , and 3 respectively.

\section{Objective}

The primary objective of the study was to determine the incidence of in-hospital AKI in COVID-19 patients and to study baseline characteristics and laboratory data associated with its development. The secondary objective of the study was in-hospital mortality associated with AKI in COVID-19 patients determined by both a univariate and an adjusted regression model.

\section{Statistical Analysis}

Continuous variables were described as mean \pm standard deviation for normally distributed data, or median and interquartile range (IQR) values for non-normal distribution. Categorical variables were presented as frequency and percentage. The mean of continuous variables was compared by using independent $t$ tests, the Wilcoxon rank sum test, and the Kruskal-Wallis test (across the stages of AKI). The proportion of categorical variables was compared by using $\chi^{2}$ test. Fischer's exact test was used when there were only limited data available. The Kaplan-Meier method (log-rank test) was used to determine the cumulative incidence of in-hospital mortality. We used the Cox proportional hazard (PH) regression to determine the association of kidney disease indicators and in-hospital mortality. The assumptions of the Cox $\mathrm{PH}$ 
Table 1. Baseline characteristics of COVID-19 patients admitted in the hospital stratified by baseline eGFR

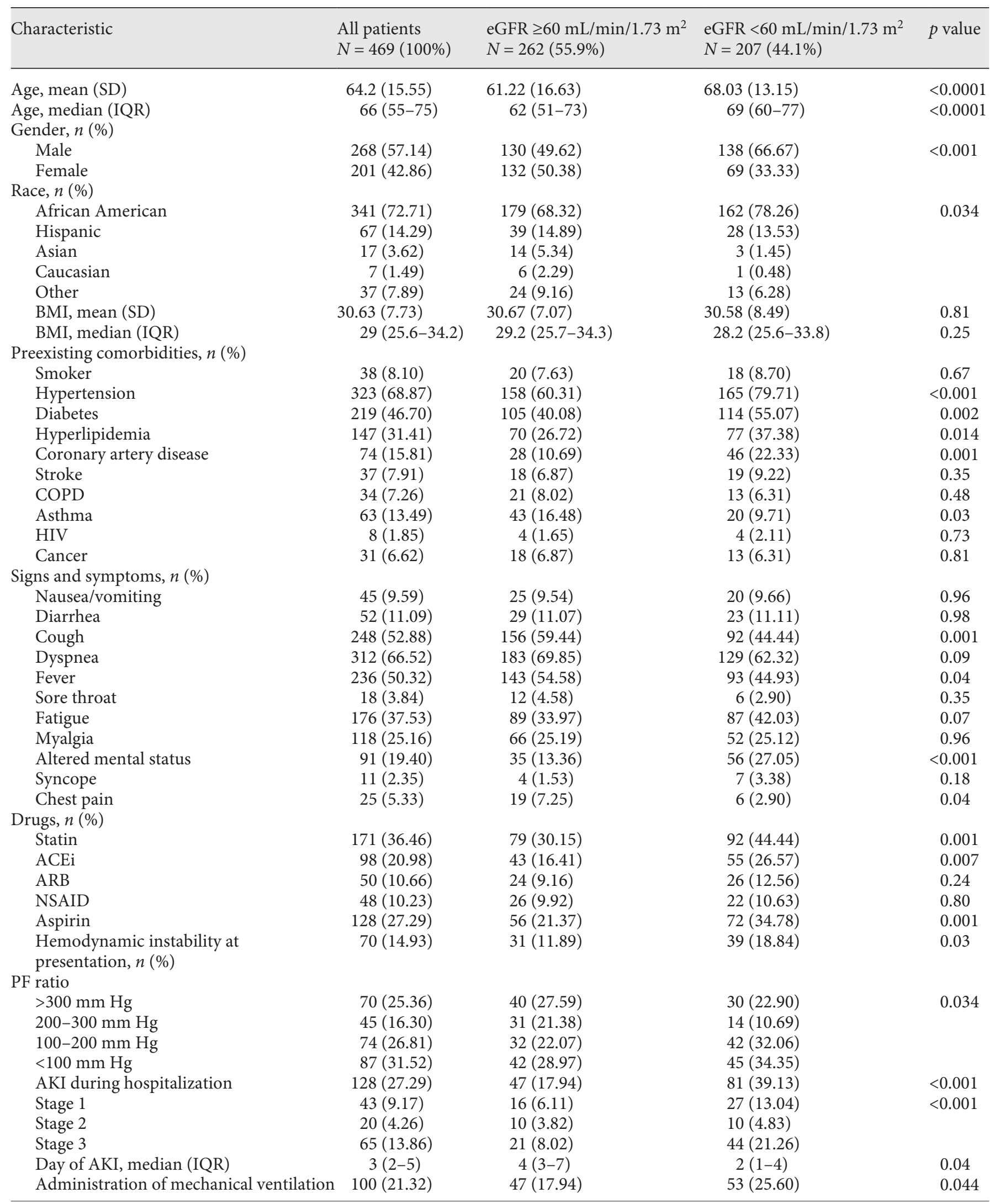


Table 1 (continued)

\begin{tabular}{lccc}
\hline Characteristic & $\begin{array}{l}\text { All patients } \\
N=469(100 \%)\end{array}$ & $\begin{array}{l}\text { eGFR } \geq 60 \mathrm{~mL} / \mathrm{min} / 1.73 \mathrm{~m}^{2} \\
N=262(55.9 \%)\end{array}$ & $\begin{array}{l}\text { eGFR }<60 \mathrm{~mL} / \mathrm{min}^{2} / .73 \mathrm{~m} \mathrm{~m}^{2} \\
N=207(44.1 \%)\end{array}$ \\
\hline Disposition on discharge, $n(\%)$ & & & $74(35.75)$ \\
Home & $228(48.61)$ & $154(58.78)$ & $11(5.31)$ \\
NH & $27(5.76)$ & $16(6.11)$ & $13(6.28)$ \\
Transferred to other hospitals & $26(5.54)$ & $13(4.96)$ & $109(52.66)$ \\
In hospital death & $188(40.09)$ & $79(30.15)$ & $7(4-10)$ \\
Length of stay in days, median (IQR) & $6.5(3-10)$ & $6(3-10)$ & 0.64 \\
\hline
\end{tabular}

PF ratio, arterial oxygen partial pressure $\left(\mathrm{PaO}_{2}\right)$ /fractional inspired oxygen $\left(\mathrm{FiO}_{2}\right)$. eGFR, estimated glomerular filtration rate; AKI, acute kidney injury; SD, standard deviation; IQR, interquartile range; BMI, basal metabolic index; COPD, chronic obstructive pulmonary disease; ACEi, angiotensin-converting enzyme inhibitor; ARB, angiotensin II receptor blockers; NSAID, non-steroidal anti-inflammatory drug; $\mathrm{NH}$, nursing home.

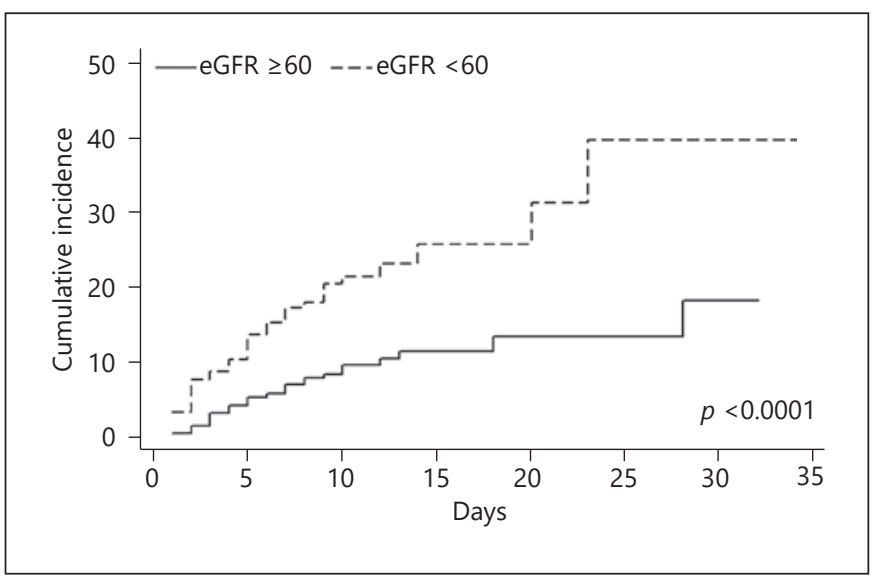

Fig. 1. Cumulative incidence of acute kidney injury in COVID-19 patients admitted in the hospital stratified by baseline admission estimated glomerular filtration rate (eGFR) in $\mathrm{mL} / \mathrm{min} / 1.73 \mathrm{~m}^{2}$.

model were checked using the Schoenfeld residuals, and no violations of the assumptions were present. In the multivariable analysis, separate $\mathrm{Cox} \mathrm{PH}$ models were used to determine the association of baseline eGFR and blood urea nitrogen (BUN) on admission, proteinuria, hematuria, and stages of AKI with in-hospital mortality since these variables showed a highly significant correlation. Variables for Cox PH regression were selected using stepwise regression. Variables with a level of risk factor $<0.10$ were entered into the model. While evaluating the association of hematuria and proteinuria with in-hospital mortality by the Cox PH model, patients with missing values $(n=256)$ were excluded from the analysis. All sta- tistical analyses were performed using the STATA version 14.2 software and a $p$ value of $<0.05$ was used to determine statistical significance.

\section{Results}

\section{Sample Size and Baseline Characteristics}

In this study, 500 adult confirmed cases of COVID-19 were initially reviewed. Patients on maintenance hemodialysis $(n=31)$ were excluded from the study and 469 patients were used for the analysis. Baseline characteristics of these patients are shown in Table $1.72 .7 \%$ of our cohort were African Americans and 14.3\% were Hispanics. The median age of the patients was 66 years (IQR $55-75)$, and $57.1 \%$ of them were male. Most of these patients presented with dyspnea (66.5\%), cough (52.9\%), and fever (50.3\%). 14.9\% of the patients had a systolic blood pressure below $100 \mathrm{~mm} \mathrm{Hg}$ at presentation. 21.3\% ( $n=100)$ of all patients were mechanically ventilated during the hospitalization. The median values of ferritin, Ddimer, hs-CRP, creatinine kinase and lactose dehydrogenase were elevated above their reference ranges (see online suppl. Table 1; for all online suppl. material, see www. karger.com/doi/10.1159/000511160). 54.3\% $(n=255)$ of all the patients studied were discharged, 5.6\% $(n=26)$ were transferred to the other institution, and $40.1 \%$ ( $n=$ 188) died during hospitalization.

\section{Renal Dysfunction}

On admission, eGFR was $<60 \mathrm{~mL} / \mathrm{min} / 1.73 \mathrm{~m}^{2}$ in $44.1 \%(n=207)$ of the patients. 70.4 and $52.5 \%$ of the patients, in which these data were available $(45.4 \%$ of the 
Table 2. Baseline characteristics of COVID-19 patients stratified by in-hospital AKI status

\begin{tabular}{|c|c|c|c|c|}
\hline Characteristics & $\begin{array}{l}\text { All patients } \\
(N=469)\end{array}$ & $\begin{array}{l}\text { No-AKI } \\
(N=341)\end{array}$ & $\begin{array}{l}\text { AKI } \\
(N=128)\end{array}$ & $p$ value \\
\hline Age, median (IQR) & $66(55-75)$ & $65(55-74)$ & $67(55-76)$ & 0.26 \\
\hline \multicolumn{5}{|l|}{ Gender, $n(\%)$} \\
\hline Male & $268(57.14)$ & $179(52.49)$ & $89(69.53)$ & \multirow[t]{2}{*}{0.001} \\
\hline Female & $201(42.86)$ & $162(47.51)$ & $39(30.47)$ & \\
\hline Hispanic & $67(14.29)$ & $50(14.66)$ & $17(13.28)$ & \multirow{4}{*}{0.15} \\
\hline Asian & $17(3.62)$ & $16(4.69)$ & $1(0.78)$ & \\
\hline Caucasian & $7(1.49)$ & $4(1.17)$ & $3(2.34)$ & \\
\hline Other & $37(7.89)$ & $29(8.50)$ & $8(6.25)$ & \\
\hline BMI, median (IQR) & $29(25.6-34.2)$ & $28.9(25.2-34.3)$ & $29.2(27.1-34.1)$ & 0.14 \\
\hline \multicolumn{5}{|l|}{ Preexisting comorbidities, $n(\%)$} \\
\hline Coronary artery disease & $74(15.81)$ & $48(14.08)$ & $26(20.47)$ & 0.09 \\
\hline Stroke & $37(7.91)$ & $25(7.33)$ & $12(9.45)$ & 0.45 \\
\hline COPD & $34(7.26)$ & $24(7.04)$ & $10(7.87)$ & 0.84 \\
\hline Asthma & $63(13.49)$ & $51(14.96)$ & $12(9.52)$ & 0.13 \\
\hline HIV & $8(1.85)$ & $6(1.94)$ & $2(1.63)$ & 0.98 \\
\hline Cancer & $31(6.62)$ & $23(6.74)$ & $8(6.30)$ & 0.98 \\
\hline CKD & $50(10.66)$ & $32(9.38)$ & $18(14.06)$ & 0.14 \\
\hline \multicolumn{5}{|l|}{ Home medications, $n(\%)$} \\
\hline Statin & $171(36.46)$ & $123(36.07)$ & $48(37.50)$ & 0.74 \\
\hline ACEi & $98(20.98)$ & $63(18.48)$ & $35(27.34)$ & 0.035 \\
\hline $\mathrm{ARB}$ & $50(10.66)$ & $33(9.68)$ & $17(13.28)$ & 0.26 \\
\hline NSAID & $48(10.23)$ & $28(8.21)$ & $20(15.63)$ & 0.02 \\
\hline \multicolumn{5}{|l|}{ Disposition on discharge, $n(\%)$} \\
\hline Discharged & $228(48.61)$ & $203(59.53)$ & $25(19.53)$ & \multirow[t]{5}{*}{$<0.001$} \\
\hline Transferred to other hospital & $26(5.54)$ & $20(5.87)$ & $6(4.69)$ & \\
\hline $\mathrm{NH} /$ shelter & $27(5.76)$ & $21(6.16)$ & $6(4.69)$ & \\
\hline In-hospital death & $188(40.09)$ & $97(28.450)$ & $91(71.09)$ & \\
\hline Length of stay, median (IQR) & $6.5(3-10)$ & $6(3-9)$ & $8(5-12)$ & \\
\hline \multicolumn{5}{|l|}{ Recovery of renal functions during hospitalization } \\
\hline Full recovery & - & - & $20(15.6)$ & - \\
\hline Partial recovery & - & - & $15(11.7)$ & - \\
\hline
\end{tabular}

PF ratio, arterial oxygen partial pressure $\left(\mathrm{PaO}_{2}\right)$ /fractional inspired oxygen $\left(\mathrm{FiO}_{2}\right)$. BMI, basal metabolic index; COPD, chronic obstructive pulmonary disease; ACEi, angiotensin-converting enzyme inhibitor; ARB, angiotensin II receptor blockers; NSAID, nonsteroidal anti-inflammatory drug; $\mathrm{NH}$, nursing home. 
Fig. 2. Cumulative incidence of in-hospital mortality in COVID-19 patient by acute kidney injury (a) and admitting estimated glomerular filtration rate (eGFR) (b). Shadows show the $95 \%$ confidence interval.

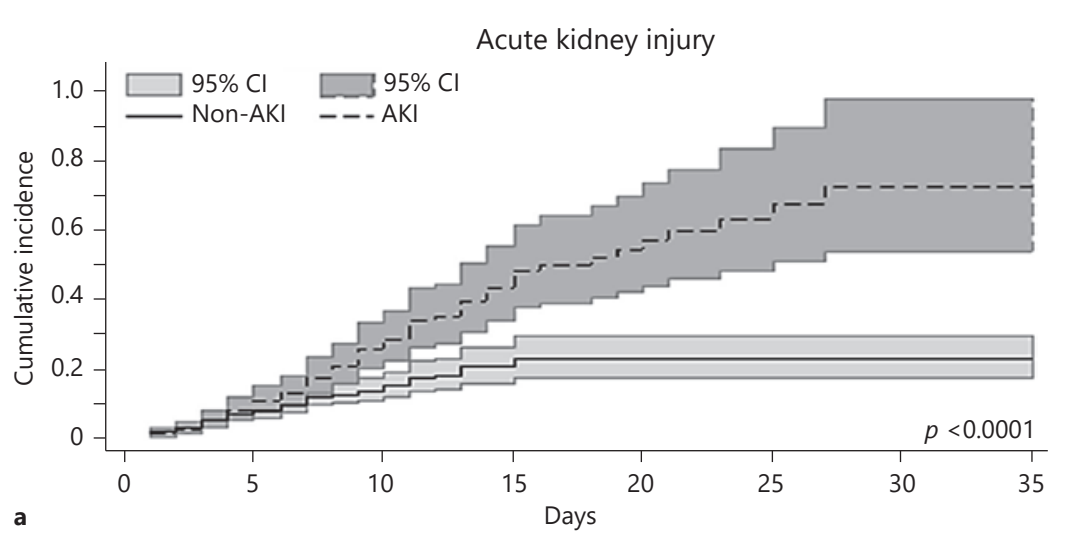

a

Baseline eGFR at admission

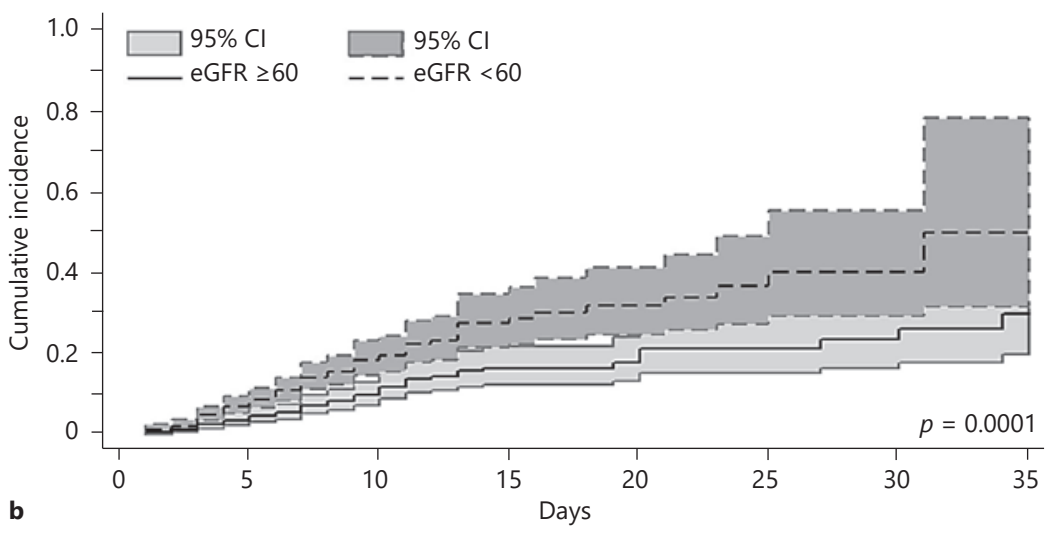

cohort), had proteinuria and hematuria, respectively. Patients with an eGFR $<60 \mathrm{~mL} / \mathrm{min} / 1.73 \mathrm{~m}^{2}$ at presentation were significantly older (median age 69 vs. 62 years; $p<$ 0.0001 ), more likely to be males (66.7 vs. $33.3 \%$ ), and have hypertension (HTN), diabetes, hyperlipidemia, and coronary artery disease among other preexisting comorbidities (Table 1). Patients with a presenting eGFR $<60 \mathrm{~mL} / \mathrm{min} / 1.73$ $\mathrm{m}^{2}$ had associated elevated ferritin, D-dimer, hs-CRP, creatinine kinase, lactate, lactate dehydrogenase, and troponin on admission $(p<0.05)$ (online suppl. Table 1$)$.

The incidence of AKI during hospitalization was $27.3 \%(n=128)$ and was significantly higher in patients who presented with eGFR $<60 \mathrm{~mL} / \mathrm{min} / 1.73 \mathrm{~m}^{2}$ (39.1 vs. 17.9\%) (Table 1; Fig. 1). 33.6, 15.6, and $50.8 \%$ of these patients had AKI stages 1, 2 and 3, respectively. The development of AKI was seen earlier in the course in patients with an eGFR $<60 \mathrm{~mL} / \mathrm{min} / 1.73 \mathrm{~m}^{2}$ at presentation $(53.1$ vs. $23.4 \%$ ) and occurred within $48 \mathrm{~h}$ of admission. Patients who developed AKI during the hospitalization were more likely to have a systolic blood pressure of $<100$ $\mathrm{mm} \mathrm{Hg}$ at presentation and a lesser PF ratio and were more likely to be intubated during the course of hospitalization (Table 2). Eighteen out of 50 patients with documented chronic kidney disease (CKD) prior to admission developed AKI during the hospitalization. Baseline laboratory values of these patients who developed AKI during hospitalization and those who did not are presented in online suppl. Table 2.

The number of patients requiring renal replacement therapy (RRT) during the hospitalization was 22 . This represented $4.7 \%$ of all patients and $17.2 \%$ of those with AKI. All the patients who required RRT by definition had stage 3 AKI. The modalities of RRT were continuous RRT in 12 patients ( $54.5 \%$ of all those who required RRT) and intermittent hemodialysis in 8 patients $(36.4 \%)$. Two patients $(9.1 \%)$ required both modalities of RRT. 68\% (68 out of 100) of those patients who required mechanical ventilation developed AKI compared with $16.3 \%$ (60 out of 369) of nonventilated patients. Of these 68 patients, 19 (27.9\%) required RRT. The majority of AKI stage 3 pa- 
tients requiring RRT were on mechanical ventilation (19 out of 22 requiring RRT). $81.8 \%$ of the patients who were treated with RRT (18 out of 22 patients) died during the hospitalization. Of those with stage 3 AKI who were not dialyzed, $90 \%$ died. Renal function recovered sufficiently in 2 patients treated with RRT and both no longer required dialysis upon discharge.

Urine studies were available for $45.5 \%$ of all patients ( $n=213$ ), and in $60.1 \%$ (77 out of 128) of those patients who developed AKI. Hematuria of $2+$ to $3+$ was found by urine dipstick in $46.8 \%$ of AKI patients, while proteinuria of $2+$ to $3+$ was detected in $53.2 \%$ of the patients with AKI.

\section{AKI and In-Hospital Mortality}

Overall, the in-hospital mortality was $40.1 \%$ in COVID-19 patients. The in-hospital mortality was significantly higher in patients with an eGFR $<60 \mathrm{~mL} / \mathrm{min} / 1.73 \mathrm{~m}^{2}$ (52.6\%) than in patients with an eGFR $\geq 60 \mathrm{~mL} / \mathrm{min} / 1.73$ $\mathrm{m}^{2}(30.2 \%)$ at presentation. The in-hospital mortality of AKI patients was $71.1 \%$ (91 out of 128 who developed AKI died) (Table 2).

A significantly higher in-hospital mortality was observed in patients with an eGFR $<60 \mathrm{~mL} / \mathrm{min} / 1.73 \mathrm{~m}^{2}$ at presentation, elevated BUN at presentation, and in-hospital AKI using the Kaplan-Meier analysis (Fig. 2). Age $\geq 75$ years (hazard ratio [HR] 2.2; $p<0.001$ ), preexisting comorbidities (diabetes mellitus [DM], HR 1.44; stroke, HR 1.97; and malignancy, HR 1.72; $p<0.05)$, $\mathrm{PF}$ ratio $<200$ $\mathrm{mm} \mathrm{Hg}$ (HR 2.3; $p<0.001)$, initial systolic blood pressure of $<100 \mathrm{~mm} \mathrm{Hg}$ (HR 2.4; $p<0.001$ ), elevated presenting BUN (HR 1.73; $p<0.001$ ), eGFR $<60 \mathrm{~mL} / \mathrm{min} / 1.73 \mathrm{~m}^{2}$ at presentation (HR 1.73; $p<0.001$ ), and in-hospital AKI stage 2 (HR 1.96; $p=0.02$ ) and stage 3 (HR 2.3; $p<0.001$ ) were all associated with a higher in-hospital mortality by univariate Cox regression analysis (online suppl. Table 3 ). In a multivariable Cox $\mathrm{PH}$ model, adjusted for age, gender, PF ratio, hemodynamic status, and comorbidities (diabetes, HTN, hyperlipidemia, cancer, and stroke), eGFR $<60 \mathrm{~mL} / \mathrm{min} / 1.73 \mathrm{~m}^{2}$ at presentation, BUN on admission, and AKI greater than or equal to stage 2 was associated with a higher risk of in-hospital mortality. There was no significant association between in-hospital mortality and proteinuria and/or hematuria (Table 4).

\section{Discussion}

AKI is common among hospitalized patients (incidence $8-22 \%)$ and is associated with increased morbidity and mortality [15-17]. In our cohort of 469 COV-
ID-19 patients, a relatively higher percentage of patients $(27.3 \%, 128$ of 469$)$ developed in-hospital AKI. Current evidence suggests that AKI in COVID-19 patients is a result of an interplay of virus-mediated injury, a dysregulated inflammatory response, angiotensin II pathway activation, hypercoagulation, and microangiopathy [18]. These COVID-19-specific factors likely interact with the other known risk factors for AKI, such as hemodynamic instability, hypoxia, and sepsis, which increased over time. The increase in the cumulative incidence of AKI over time in our cohort is likely associated with concomitant clinical deterioration and less likely represent a unique renal effect of COVID-19. Compared to studies of AKI in COVID-19 patients from other countries, we found our incidence to be higher than that reported from 2 Asian countries (0-9.2\%), and is similar to that in an Italian study (27.8\%) [5-9]. However, it is lower than that in the more recent report by Hirsch et al. [4] who detailed their experience in 13 academic and community hospitals in metropolitan New York (36.6\%), none of which are located in the inner city, lower socioeconomic neighborhoods with a predominant minority population. As compared with the above-cited studies, the incidence of stage 3 AKI (13.9\%) was highest in our cohort. It is not possible to determine with certainty the cause(s) of this wide variation. However, we did note a higher prevalence of comorbid conditions in our group. For example, Cheng et al. [5] reported a $5.1 \%$ incidence of AKI, with a $33.4 \%$ prevalence of HTN and a $14.3 \%$ prevalence of DM in their study population. In our cohort, roughly twice as many (68.9\%) had HTN, and more than 3 times as many $(46.7 \%)$ were diabetic (Table 1). Also, the incidence of severe lung involvement that required mechanical ventilation was lower (13.4\%) than ours (21.3\%) (Table 1). Mechanical ventilation per se has been reported to be strongly associated with AKI [4]. In our cohort, the incidence of AKI was also higher among those requiring mechanical ventilation $(68 \%)$.

We identified various risk factors for AKI in our study (Tables 2,3). Similar to previous reports, our patients with AKI were more likely to be male, have a history of HTN, and have severe respiratory failure requiring mechanical ventilation $[4,5]$. In our study, higher BMI increased the likelihood of developing severe AKI. A low eGFR at presentation more than doubled the risk of developing AKI $(p<0.001)$ (Table 1). Patients with lower eGFR had a significantly higher cumulative incidence of AKI than those with eGFR $>60 \mathrm{~mL} / \mathrm{min} / 1.73 \mathrm{~m}^{2}(p<$ 0.001 ; Fig. 1). Thus, there was a synergistic effect of reduced baseline kidney function and subsequent AKI. We 


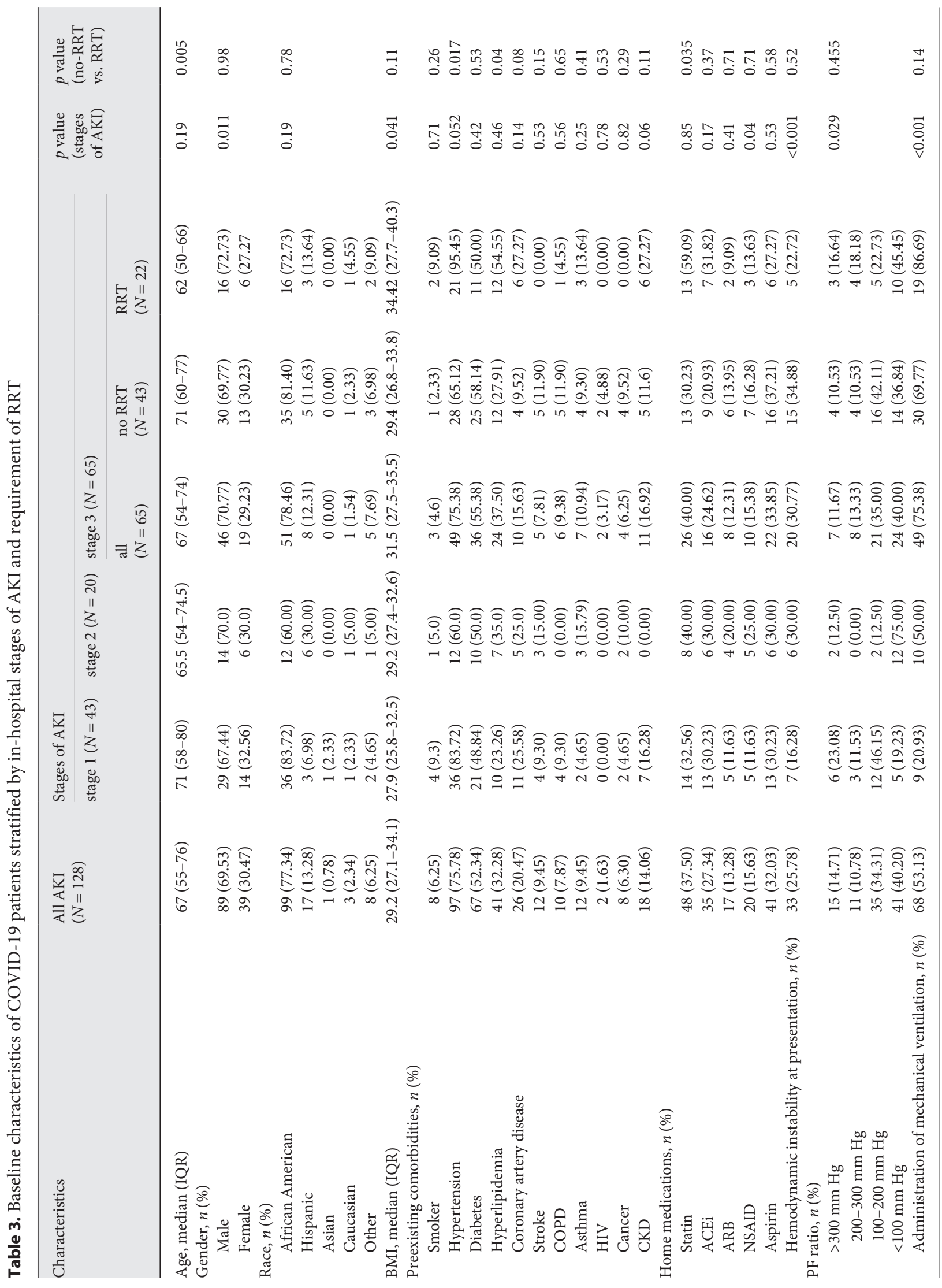




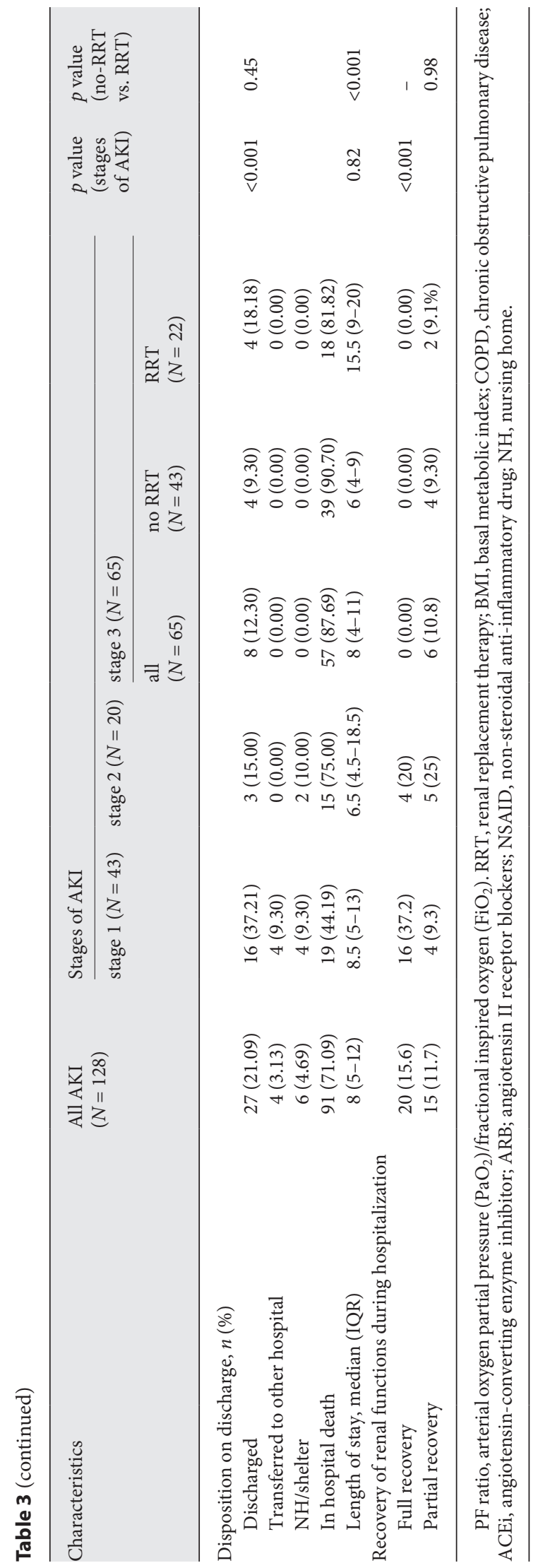

Table 4. Association of kidney disease with 30-day in-hospital mortality in COVID-19 patients using adjusted ${ }^{\text {a }}$ proportional hazard model

\begin{tabular}{llll}
\hline Variables & $\begin{array}{l}\text { Hazard } \\
\text { ratios }\end{array}$ & 95\% CI & $\begin{array}{l}p \\
\text { value }^{\mathrm{b}}\end{array}$ \\
\hline Baseline eGFR $<60 \mathrm{~mL} / \mathrm{min} / 1.73 \mathrm{~m}^{2}$ & 1.43 & $1.1-2.03$ & 0.04 \\
Acute kidney injury & 1.8 & $1.22-2.35$ & 0.002 \\
Stage 1 & 1.14 & $0.64-2.03$ & 0.64 \\
Stage 2 & 1.86 & $1.03-3.56$ & 0.044 \\
Stage 3 & 2.1 & $1.30-2.81$ & 0.001 \\
\hline
\end{tabular}

a Adjusted for age, gender, PF ratio, hemodynamic status at baseline, and comorbidities (diabetes, hypertension, hyperlipidemia, cancer, and stroke). ${ }^{\mathrm{b}}$ Separate Cox proportional hazard models were run as of strong correlation among these variables.

did not find an association between diabetes and in-hospital AKI.

The role of angiotensin-converting enzyme inhibitors (ACEi) in COVID-19 is a subject of ongoing debate. ACEi can increase the expression of ACE2, the receptor used by SARS-CoV-2 to enter target cells $[19,20]$. However, ACE2 has been shown to be beneficial in acute respiratory distress syndrome when replaced $[21,22]$. This possible protective effect is attributed to the degradation of proinflammatory angiotensin II to angiotensin by ACE2. At present, it remains unclear if ACEi facilitate severe acute respiratory syndrome coronavirus 2 (SARS-CoV-2) cellular entry and intracellular virus replication and whether ACEi improve acute respiratory distress syndrome outcomes. In our cohort, the use of ACEi was significantly higher in patients with a lower admitting eGFR (Table 1) and in those who experienced AKI (Table 2). We found a similar association between AKI and nonsteroidal anti-inflammatory drugs, but not with angiotensin II receptor blockers (ARBs) in our cohort (Table 2). Further studies are necessary to delineate the relationship between ACEi, angiotensin II receptor blockers, and COVID-19 outcomes.

Markers of acute inflammation (ferritin and CRP), muscle injury (creatinine kinase), myocardial injury, and D-dimer levels were significantly higher in patients with a low eGFR at presentation (online suppl. Table 1) and in those with AKI (online suppl. Table 2), suggesting causality by a dysregulated immune/inflammatory response, the so-called "cytokine storm."

We found an extremely high incidence of mortality in our study cohort. High presenting BUN and low eGFR $\left(<60 \mathrm{~mL} / \mathrm{min} / 1.73 \mathrm{~m}^{2}\right)$ were significantly associated with 
increased mortality (Table 1). AKI was significantly associated with increased mortality. The mortality risk was highest in stage 3 AKI (HR 2.1; CI 1.3-2.81) (Table 4). Mortality was $71.1 \%$ in the AKI group and $28.5 \%$ in the non-AKI group $(p<0.001)$. Mortality rose concurrently with the AKI stages, $44.2,75$, and $87.7 \%$ for stages 1,2 , and 3 , respectively (Table 3 ). These rates were over 2 - and 5 -fold higher than those reported by Hirsch et al. [4] (34.8\% AKI, $5.6 \%$ in non-AKI). However, our cohort's high mortality is similar to the findings of John Hopkins University and the American Community Survey. They reported a 3- and 6-fold higher COVID-19 infection and mortality rate in predominantly African American counties than in predominantly white counties [2]. Wadhera et al. [23] also reported a death rate of 181 per 100,000 in Brooklyn, about $50 \%$ higher than that of Manhattan. Moreover, in New York City, current age-adjusted mortality is highest among African Americans and about 1.5 times that of whites [24].

Our hospital provides medical services for an ethnic minority and a low-income population in the most impoverished neighborhood in Brooklyn, where $37 \%$ of the residents live below the Federal Poverty Level as per a 2015 report [25]. 72.7\% of our patients identified themselves as African Americans. In comparison, in the study by Hirsch et al. [4], 38.8\% identified themselves as whites, and only $20.6 \%$ identified themselves as African Americans. The high representation of ethnic minorities in our study population may have contributed to the increased mortality. This association has been widely publicized and is likely the result of a complex interplay of multiple factors. These include, but are not limited to, a high burden of comorbidities, genetics, the pernicious influence of adverse socioeconomic determinants of health, the absence of the privilege to work from home, and the ability to follow the recommended social distancing [3].

In our cohort, 22 (17.2\%) of the 128 patients with AKI required RRT. Nineteen out of these 22 patients (86.4\%) were mechanically ventilated. Thus, almost all of the additional requirements for acute dialysis during the $\mathrm{CO}$ VID-19 pandemic at our institution were in intensive care units. Overall, 27.9\% (19 out of 68) of mechanically ventilated patients required RRT. Elevated ferritin was associated with an increased risk of all stages of AKI and the need for RRT (online suppl. Table 2). Among those who received RRT, 18 (81.8\%) died versus 39 (90.7\%) in the stage 3 AKI group who did not undergo RRT ( $p=$ 0.45 ) (Table 3 ). Thus, there was no statistically significant improvement in survival, and the mortality remained extremely high in both the RRT and non-RRT groups. These findings should prompt the early involvement of a palliative care team to define goals of care in patients with COVID-19 disease and stage 3 AKI.

In our experience, ultrafiltration was not well tolerated. It often increased pressor requirements and did little to improve the ventilatory status. We observed a high incidence of hypercoagulability and frequent clotting of the dialysis filters, lines, and catheters. Thus, the patients required higher than the usual doses of heparin.

Our study has limitations. Although 207 (44.1\%) of the study population had a low eGFR on admission, only 50 (10.7\%) had a documented history of CKD. Data were collected retrospectively from medical records and often did not have information regarding prior renal function. Patients also may have had an elevated creatinine on admission secondary to AKI suffered prior to hospitalization. If so, we may have underestimated the true overall incidence of COVID-19-associated AKI. It is not possible to rectify this problem, given the data at our disposal.

Our study is a single-center study, with the majority of the study population identifying themselves as African Americans or Hispanics. This limits our study's general applicability but provides a unique insight into this population and broadens our understanding of COVID-19 disease. Data were missing on many patients at different stages of the hospitalization since they were collected retrospectively. Importantly, little data were available regarding urine output, a defining characteristic of AKI. With this lack of information, we may have again underestimated the true incidence of AKI.

Nevertheless, even with the above limitations of our study, the high incidence of AKI and its negative impact upon survival is abundantly clear. In response to the high incidence of AKI in our patients with COVID-19, we increased our capacity to deliver RRT. These efforts included the purchase of additional machines and dialysis solutions, additional staff training, and engineering alterations to allow dialysis on the general medical floors. Also, we increased our stockpiles of catheters, filters, and dialysis solutions for intermittent and continuous RRT. We also plan to establish a peritoneal dialysis program to further strengthen our acute dialysis capacity.

In conclusion, our data report explicitly upon a high proportion of ethnic minority patients in an inner city, severely economically deprived neighborhood, previously not studied. Our patients experienced a high incidence of AKI and an extremely high mortality, especially in AKI stage 3. Unfortunately, RRT provided little survival benefit. Policy changes and planning in preparation for this high incidence of AKI in COVID-19 patients are necessary at the local and national levels. 


\section{Statement of Ethics}

Study was approved by Institutional Review Board (IRB), Brookdale Hospital.

\section{Conflict of Interest Statement}

The authors have no conflicts of interests to declare.

\section{Funding Sources}

The authors did not receive any funding.

\section{Author Contributions}

U.Z. - wrote manuscript and literature review; P.R. - IRB approval and data and manuscript review; S.S., L.A., P.K. - manuscript review; A.C., M.A., G.M., A.S., and J.T.N. - data collection; and P.B. - supervisor

\section{References}

1 Huang C, Wang Y, Li X, Ren L, Zhao J, Hu Y, et al. Clinical features of patients infected with 2019 novel coronavirus in Wuhan, China. Lancet. 2020;395(10223):497-506.

2 Thebault R, Ba Tran A, Williams V. The coronavirus is infecting and killing black Americans at an alarmingly high rate. Washington Post. 2020 Apr 7. Available from: https:// www.washingtonpost.com/nation/2020/04/ 07/coronavirus-is-infecting-killing-blackamericans-an-alarmingly-high-rate-postanalysis-shows/.

3 Yancy CW. COVID-19 and African Americans. JAMA. 2020;323(19):1891-2.

4 Hirsch JS, Ng JH, Ross DW, Sharma P, Shah $\mathrm{HH}$, Barnett RL, et al. Acute kidney injury in patients hospitalized with COVID-19. kidney Int. 2020 Jul;98(1):209-18.

5 Cheng Y, Luo R, Wang K, Zhang M, Wang Z, Dong $\mathrm{L}$, et al. Kidney disease is associated with in-hospital death of patients with COVID-19. Kidney Int. 2020;97(5):829-38.

6 Wang L, Li X, Chen H, Yan S, Li D, Li Y. Coronavirus disease 19 infection does not result in acute kidney injury: an analysis of 116 hospitalized patients from Wuhan, China. Am J Nephrol. 2020;51(5):343-8.

7 Wang D, Hu B, Hu C, Zhu F, Liu X, Zhang J, et al. Clinical characteristics of 138 hospitalized patients with 2019 novel coronavirus-infected pneumonia in Wuhan, China. JAMA. 2020;323(11):1061.

8 Hong KS, Lee KH, Chung JH, Shin KC, Choi $\mathrm{EY}$, Jin HJ, et al. Clinical features and outcomes of 98 patients hospitalized with SARSCoV-2 infection in Daegu, South Korea: a brief descriptive study. Yonsei Med J. 2020 May;61(5):431-7.
9 Fanelli V, Fiorentino M, Cantaluppi V, Gesualdo L, Stallone G, Ronco C, et al. Acute kidney injury in SARS-CoV-2 infected patients. Crit Care. 2020;24(1):155.

10 Hoffmann M, Kleine-Weber H, Krüger N, Müller M, Drosten C, Pöhlmann S. The novel coronavirus 2019 (2019-nCoV) uses the SARS-coronavirus receptor ACE2 and the cellular protease TMPRSS2 for entry into target cells. bioRxiv. 2020. Posted 2020 Jan 31.

11 Zhao Y, Zhao Z, Wang Y, Zhou Y, Ma Y, Zuo W. Single-cell RNA expression profiling of ACE2, the putative receptor of Wuhan 2019 nCov. bioRxiv. 2020.

12 Hamming I, Timens W, Bulthuis ML, Lely AT, Navis G, van Goor H. Tissue distribution of ACE2 protein, the functional receptor for SARS coronavirus. A first step in understanding SARS pathogenesis. J Pathol. 2004;203(2): 631.

13 Su H, Yang M, Wan C, Yi LX, Tang F, Zhu HY. Renal histopathological analysis of 26 postmortem findings of patients with $\mathrm{COV}$ ID-19 in China. Kidney Int. 2020 Jul;98(1): 219-27.

14 Khwaja A. KDIGO clinical practice guidelines for acute kidney injury. Nephron Clin Pract. 2012;120(4):c179-84.

15 Khadzhynov D, Schmidt D, Hardt J, Rauch G, Gocke P, Eckardt KU, et al. The incidence of acute kidney injury and associated hospital mortality. Dtsch Arztebl Int. 2019;116(22): 397-404

16 Sawhney S, Marks A, Fluck N, Levin A, Prescott $\mathrm{G}$, Black C. Intermediate and long-term outcomes of survivors of acute kidney injury episodes: a large population-based cohort study. Am J Kidney Dis. 2017;69(1):18-28.
17 Wang HE, Muntner P, Chertow GM, Warnock DG. Acute kidney injury and mortality in hospitalized patients. Am J Nephrol. 2012; 35(4):349-55.

18 Batlle D, Soler MJ, Sparks MA, Hiremath S, South AM, Welling P, et al. Acute kidney injury in COVID-19: emerging evidence of a distinct pathophysiology. J Am Soc Nephrol. $2020 \mathrm{Jul} ; 31(7): 1380-3$.

19 Ferrario CM, Jessup J, Chappell MC, Averill DB, Brosnihan KB, Tallant EA, et al. Effect of angiotensin-converting enzyme inhibition and angiotensin II receptor blockers on cardiac angiotensin-converting enzyme 2 . Circulation. 2005;111(20):2605-10.

20 Vuille-dit-Bille RN, Camargo SM, Emmenegger L, Sasse T, Kummer E, Jando J, et al. Human intestine luminal ACE2 and amino acid transporter expression increased by ACE-inhibitors. Amino Acids. 2015;47:693-705.

21 Kuba K, Imai Y, Rao S, Gao H, Guo F, Guan $\mathrm{B}$, et al. A crucial role of angiotensin converting enzyme 2 (ACE2) in SARS coronavirus induced lung injury. Nat Med. 2005;11: 875-9.

$22 \mathrm{Gu} \mathrm{H}$, Xie Z, Li T, Zhang S, Lai C, Zhu P, et al Angiotensin-converting enzyme 2 inhibits lung injury induced by respiratory syncytial virus. Sci Rep. 2016;6:19840

23 Wadhera RK, Wadhera P, Gaba P, Figueroa JF, Joynt Maddox KE, Yeh RW, et al. Variation in COVID-19 hospitalizations and deaths across New York city boroughs. JAMA. 2020;323(21):2192-5.

24 NYC Health. COVID-19: data. Accessed 2020 May 7. Available from: https://wwwl.nyc. gov/site/doh/covid/covid-19-data.page.

25 King L, Hinterland K, Dragan KL, Driver CR, Harris TG, Gwynn RC, et al. Community health profiles 2015. Brooklyn Community District 16: Brownsville. 2015;40(59):1-16. 